\title{
Blood Pressure Measurement and Management Telemedicine System Based on a Smart-Phone
}

\author{
http://dx.doi.org/10.3991/ijoe.v9i5.2697 \\ Asharf Tahat ${ }^{1}$, Yazeed Kheetan ${ }^{2}$, and Anwar Sacca ${ }^{3}$ \\ ${ }^{1}$ Princess Sumaya University for Technology, Amman, Jordan \\ ${ }^{2}$ Ericsson, Amman, Jordan \\ ${ }^{3}$ Elite Technologies JLT, Dubai, UAE
}

\begin{abstract}
Variation of blood pressure throughout the day is one of the reasons why it is increasingly evident that the traditional way of measuring blood pressure in the clinic or office frequently produces numbers that grossly overestimate patient's true blood pressure level. This is a major problem, since it is one of the most important and frequent measurements made by physicians. High blood pressure (BP) (hypertension) is a leading chronic condition in the globe and a major risk factor for severe diseases. Measuring the BP as accurately as possible can save a lot of human lives. However, the measurement and management platform can still be improved. In this paper, we describe a complete low-cost prototype system that we have developed for this purpose that can be part of and m-Health platform. Our BP telemedicine device is based on the oscillometric method for measuring BP. A microcontroller oversees measurement operations, to process acquired readings, and to calculate the heart-rate. A smart mobile phone commands the operation of our developed system via Bluetooth. A custom Windows Mobile application software was designed and developed to command the operation of this platform and to receive these vital measurements in a convenient manner. Obtained measurements can be stored on the mobile device to form a local database or transmitted via a designated wireless protocol.
\end{abstract}

Index Terms-m-health; telemedicine; blood pressure; pervasive computing; Bluetooth; wireless vital signs monitoring.

\section{INTRODUCTION}

Blood is carried from the heart to all parts of human body in vessels called arteries. Blood pressure is the force of the blood pushing against the walls of the arteries. Each time the heart beats (about 60-70 times a minute at rest) it pumps out blood into the arteries. Blood pressure (BP), defined as the pressure exerted by circulating blood upon the walls of blood vessels, is an important biomarker of cardiovascular health, with different value of systolic blood pressure SBP (highest blood pressure when the heart beats) and different value of diastolic blood pressure DBP (lowest blood pressure when the heart relaxes). Values of SBP and DBP change throughout the day with dependence on person's physical and psychical activity. According to a World Health Organization (WHO) estimate, raised blood pressure (BP) is estimated to cause 7.1 million deaths, about $13 \%$ of the global total [1]. High BP (Hypertension) is the most common chronic medical problem prompting visits to primary health care providers, almost 1 billion people have BP worldwide, and more than a half a billion more will acquire this silent killer by
2025 [2]. Uncontrolled hypertension is an ongoing challenge to health care systems worldwide. Proper measurement, management, and interpretation of the BP are essential in the diagnosis and management of hypertension [3]. One of the most common medical instruments used at home is the automatic non-invasive BP meter. Automated oscillometric blood pressure devices have been used extensively on a daily basis for self-based monitoring scenarios. The oscillometric BP measurement principle relies on following the oscillations in the cuff pressure that is wrapped around the upper arm of a patient [4].

Telemedicine and m-Health are widely considered to be part of the inevitable future of the modern practice of medicine. It is gaining more momentum as a new approach for patients' surveillance outside of hospitals (at home) to facilitate early diagnosis, treatment, and for increased convenience. Telemedicine is currently being used by doctors, hospitals, and other health care providers around the world [5]. The mobile phone has been recognized as a possible tool for telemedicine since it became commercially available [6]. Currently, there are 6.8 billion mobile phone users in the world, and it is estimated that $77 \%$ of them are in developing countries [7], while it is anticipated that there will be more mobile phones in the world than there are humans.

Moreover, newer cellular access technologies, such as 3G, HSDPA/HSUPA, LTE, in addition to WiFi hotspots provide for much higher data transmission speeds (rates) than basic $2 \mathrm{G}$ cellular systems that already cover about $90 \%$ of the world area [7], which offers future telemedicine solutions endless choices for high-end designs.

The proposed mobile blood pressure (monitoring) system is shown in Fig. 1 [8]. The patient (client) and the health care professional (server) can be located anywhere in the globe where there is wireless (cellular) network coverage. The patient's BP and heart-rate (HR) can be acquired by the patient himself, or the patient can be assisted by a family member or a health care professional in more serious cases depending on the particular patient's case. The signal acquisition process is performed by wrapping the pneumatic system (cuff) around the patient's arm at a designated place as is normally done in a typical similar set-up. The client unit communicates with the mobile phone through a Bluetooth transceiver. The mobile phone application software can initiate the measurement process when instructed by the user via software interface menu. When blood pressure 


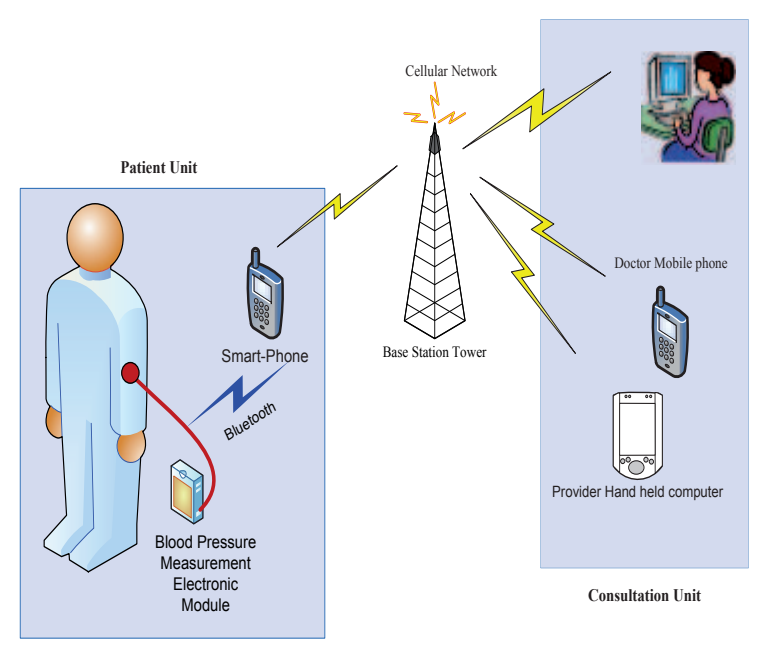

Figure. 1. Mobile BP measurement and monitoring telemedicine system

and HR measurements are ready, they are displayed on the smartphone's screen and in-turn stored or transmitted to a desired remote destination for consultation purposes or storage in a personal database .

\section{The BP MEASUREMENT UNIT}

Our device deploys the oscillometric blood pressure measurement method [3], [4]. The oscillometric method operates by sensing the magnitude of oscillations caused by the blood as it begins to flow again into the limb after it had been occluded during the full inflation of the cuff. This method of measuring blood pressure is very often used for the measurement of the blood pressure because of its excellent reliability.

In our design we created a blood pressure monitoring device that measures the blood pressure using a microcontroller (PIC16F877A), a pressure sensor MPX5050GP, and a Bluetooth module (LinkMatik 2.0). The microcontroller controls a valve and an air pump. These were integrated on custom-made printed circuit board (PCB) as shown in Fig. 2. Also, Fig. 3 is a block diagram that depicts interconnection of the device's components. When a measure command is received via Bluetooth from the mobile interface, the microcontroller will close the valve and trigger the air pump to inflate the cuff to a certain predefined high pressure value.

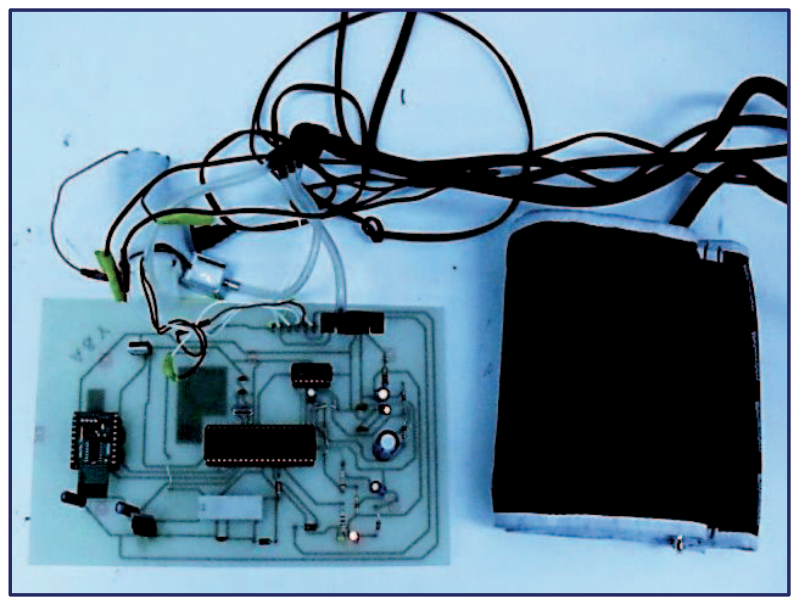

Figure 2. PCB of our blood pressure monitor device.

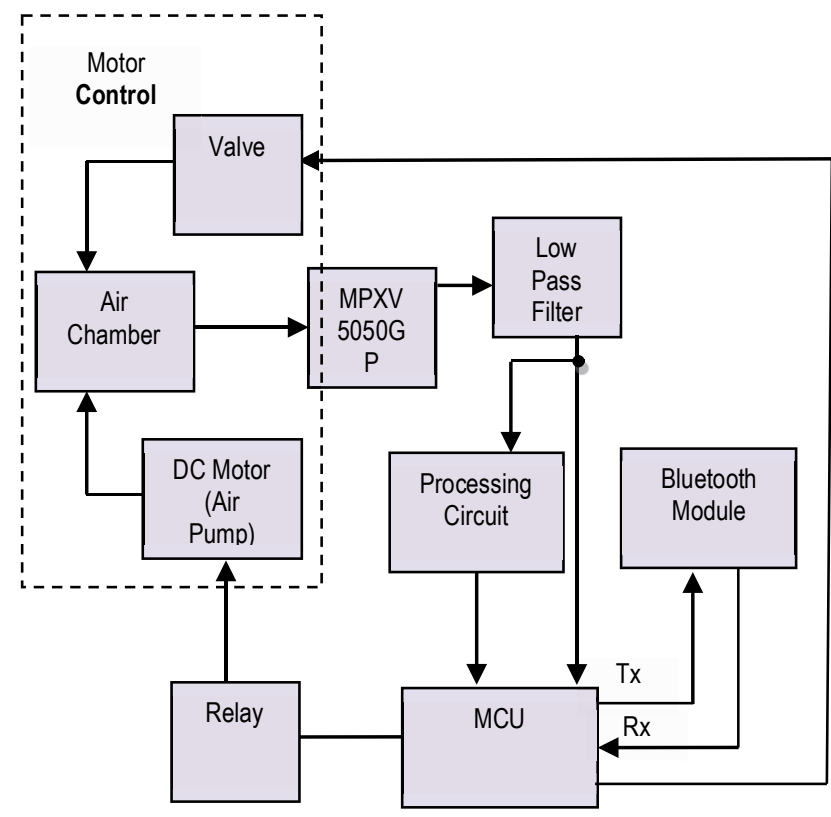

Figure 3. A block diagram of the BP measurement hardware unit of the system.

Then the cuff will start to deflate slowly through the bleeding valve. The microcontroller will also acquire the sensor's measurement through its analog-to-digital converter $(\mathrm{A} / \mathrm{D})$ module. It will also calculate the $\mathrm{BP}$ and $\mathrm{HR}$ based on these measurements. When readings are ready, the microcontroller will open the valve and send the acquired readings via the Bluetooth module to the controlling mobile phone.

\section{A. Air-Pump and Vlave Control}

An output signal from the microcontroller should close the valve, and another signal should be able to turn-on the air pump. The solenoid valve is a normally opened. In order to close the valve and trap the air inside the air chamber (cuff), the microcontroller should send a high signal to the valve. This can be done directly because the microcontroller's output voltage is sufficient to close the valve. The process of interfacing the air pump to the microcontroller is a little more complicated than that of the valve. That is because the air pump is an inductive load (has a motor in it), thus it draws large amount of current; this implies that the microcontroller's output voltage/current will not be sufficient enough to turn on the motor. A relay was used to turn on the motor.

\section{B. Analog Pressure Sensor Output Filterings}

The pressure sensor MPX5050GP [9], which is an integrated pressure sensor, has trimmed outputs, built-in temperature compensation and an amplified single-ended output which make it compatible with ADC of our microcontroller. This sensor has a built-in on chip differential amplifier that level shifts and amplifies the very small differential voltage produced by the transducer bridge, translating this voltage to a single-ended output voltage ranging from 0.2 to 4.7 volts.

The two dominant types of noise in a piezo-resistive integrated pressure sensor are shot (white) noise and $1 / f$ (flicker noise). Noise can also come from external 
circuits. Therefore, power supply, grounding and PCB layout is important and needs special consideration. Although the transducer has a response of about $500 \mathrm{~Hz}$, its noise output extends from $500 \mathrm{~Hz}$ to $1 \mathrm{MHz}$. To solve this problem we used decoupling capacitor for the sensor's power supply input and hardware lowpass filtering. This LPF has a cut-off frequency of approximately $650 \mathrm{~Hz}$, thus omitting the white noise occurring at higher frequencies.

\section{Processing Circuit and Interfacing to Microcontroller}

After removing the white noise riding on the sensors output using the LPF, the LPF output, which we will refer to as the sensors output for simplicity, was split into two for two different purposes. The first, which we will refer to as the raw output, is used as the cuff pressure. The second, which we will refer to as processed output, is further processed by a circuit that will be explained below.

Since MPXV5050GP is signal-conditioned, the cuff pressure can be directly interfaced with an analog-todigital (A/D) converter for digitization. So the raw output which will be used to measure the cuff pressure is connected to the PIC16F877A microcontroller analog input channel-0 (AN0). The raw output provides a voltage ranging from 0.2 to 4.7 volts which is internally converted by the microcontroller to an 8-bit unsigned integer.

The other path (the one with the processed output) will filter and amplify the raw cuff pressure (CP) signal to extract an amplified version of the CP oscillations, which are caused by the expansion of the subject's arm each time pressure in the arm increases during cardiac systole. The output of the sensor consists of two signals; the oscillation signal $(\approx 1 \mathrm{~Hz})$ riding on the $\mathrm{CP}$ signal $(\leq$ $0.04 \mathrm{~Hz}$ ). Hence, a 2-pole high pass filter is designed to block the CP signal before the amplification of the oscillation signal. If the CP signal is not properly attenuated, the baseline of the oscillation will not be constant and the amplitude of each oscillation will not have the same reference for comparison.

The oscillation signal varies from person to person. In general, it varies from less than $1 \mathrm{mmHg}$ to $3 \mathrm{mmHg}$. From the transfer function of MPXV5050GP, this will translate to a voltage output signal of $12 \mathrm{mV}$ to $36 \mathrm{mV}$. Since the filter gives an attenuation of $10 \mathrm{~dB}$ to the $1 \mathrm{~Hz}$ signal, the oscillation signal becomes $3.8 \mathrm{mV}$ to $11.4 \mathrm{mV}$ respectively. As it was indicated by the manufacturer application note (AN1571) [10], the amplification factor of the amplifier is chosen to be 150 for best results, so that the amplified oscillation signal is within the output limit of the amplifier (5.0 $\mathrm{mV}$ to $3.5 \mathrm{~V})$.

Finally, in order for the PIC16F977A to observe and make use of the oscillation signal in the measurement process, the output of this circuit (processed output) was connected to its analog to digital channel-1 (AN1).

\section{Bluetooth Tranceiver}

Bluetooth is an industrial specification for wireless Personal Area Networks (PANs) [11]. Bluetooth provides a way to connect and exchange information between devices such as mobile phones, laptops, PCs, printers, digital cameras, and video game consoles over a secure, globally unlicensed short-range radio frequency $(2.45$ $\mathrm{GHz}$ ). The Bluetooth specifications are developed and licensed by the Bluetooth Special Interest Group. It is a standard communications protocol primarily designed for low power consumption, with a short range ( 1 meter $(0 \mathrm{dBm}), 10$ meter $(4 \mathrm{dBm})$, and 100 meter $(20 \mathrm{dBm}))$.

We used the LinkMatik 2.0 Bluetooth transceiver module, which is a class 1 model that has an approximate range of 100 meters. The asynchronous data from the PIC16F877 microcontroller is delivered to the LinkMatik Bluetooth module on the serial port at a speed of 9600 bps. The Bluetooth module is configured as a Slave and the mobile phone is considered to be functioning as a Master. The microcontroller sends/receives data to/from the Bluetooth module, which transmits/receives data continuously as raw binary bytes.

The Bluetooth standard aims at guaranteeing reliability and robustness with acceptable security that stems from the fact that it uses Frequency Hopped Spread Spectrum (FHSS) technique, which generated an increased demand for applying Bluetooth technology in the healthcare world [12]. Also, Bluetooth requires authentication to restrict connectivity to devices when configured in security modes. Additionally, it uses encryption to employ secret keys where only authorized users can make data intelligible again

\section{The BP MeAsurement Process}

The oscillometric method operates by sensing the magnitude of oscillations caused by the blood as it begins to flow again into the limb after it had been occluded during the full inflation of the cuff [4]. When the pressure decreases in the cuff, the artery starts to emit pulsations; the pressure then measured on the device defines the maximal blood pressure or Systolic blood pressure (SBP). During the pressure decrease in the cuff, the oscillations will become increasingly significant, until maximum amplitude of these oscillations defines the average blood pressure or mean arterial pressure (MAP). Then, the oscillations can still be seen during the decrease of the pressure in the cuff, until they disappear; the pressure then read on the device defines the minimal blood pressure or Diastolic blood pressure (DBP). This method of measuring blood pressure is very often used for the measurement of the blood pressure because of its excellent reliability.

\section{A. Systolic Pressure Measurement Method}

After the motor pumps the pressure up to $180 \mathrm{mmHg}$ which is approximately more than the systolic pressure of normal healthy people, the cuff starts deflating and the program enters systolic measure mode. In this mode, the program will look at the AC waveform from AN0 pin (processed sensor output that represents the tiny oscillations on the cuff). When the pressure in the cuff decreases to a certain value, the blood begins to flow through the arm. At this time if we look at the oscilloscope, we will see the onset of the oscillation as shown in Fig. 4 [10]. The systolic pressure can be obtained at this point. 


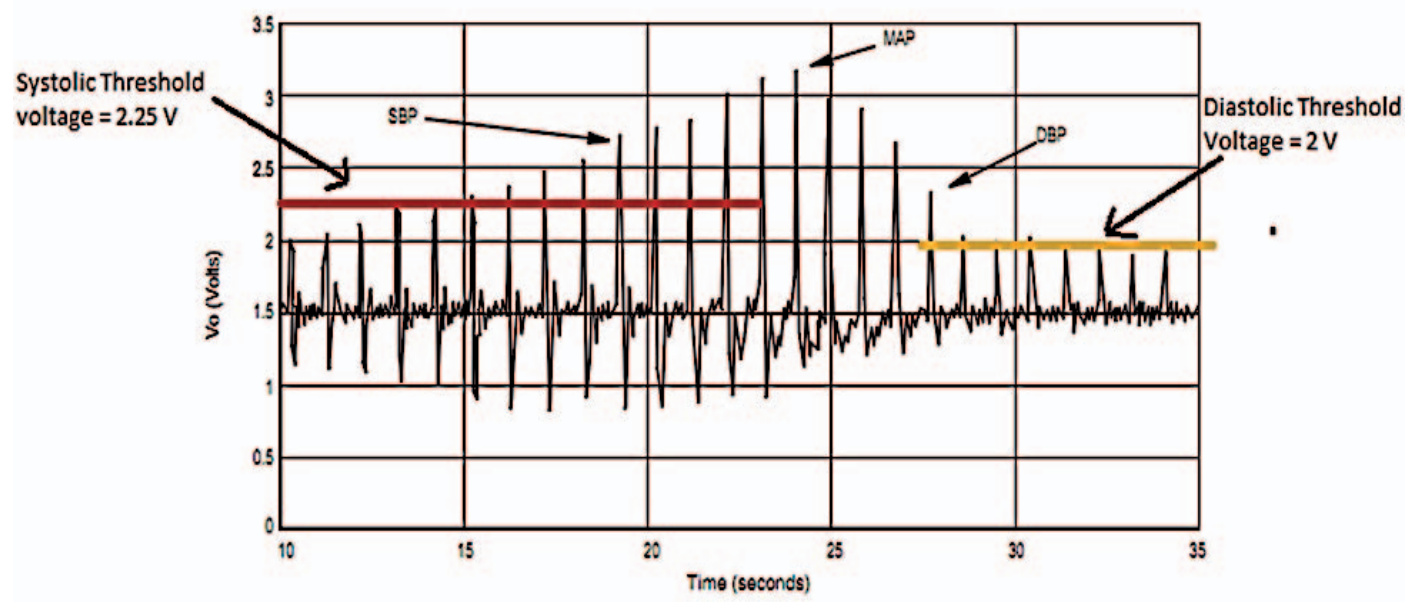

Figure 4. Illustration of threshold voltages on an extracted oscillation signal

The way this is implemented in the program is that we first set a threshold voltage of $2.25 \mathrm{~V}$ for the AC wave-form. At the start, there is no pulse and the voltage at the AN0 pin is constant at approximately $1.5 \mathrm{~V}$. When the pressure in the cuff decreases until it reaches the systolic pressure value, the oscillation starts and grows. We then count the number of pulses that has maximum values above the threshold voltage. If the program counts up to 4, the program records the DC voltage from pin AN1 (sensor raw output that indicates the cuff pressure). This value is equal to the systolic pressure in terms of ADC counts. We let the program count up to 4 to make sure that oscillation crossing the threshold is due to blood flow, and not due to noise or immature pulse. Once the systolic pressure value is recorded in terms of ADC counts this mode will end.

\section{B. Heart-Rate Measurement Method}

In this mode, we will measure the heart-rate (HR) of the patient. It is designed to work right after the program finishes calculating the systolic pressure. We choose to determine the pulse rate right after determining the systolic pressure because at this point the oscillation of the waveform is strongest.
The main idea here is that we want to measure the period of the oscillation (1 pulse). To accomplish this; the program samples the AC waveform every 40 millisecond. It then records the time interval between the times at which the values of the AC waveform cross the voltage value of 1.75 volts. The threshold level of valid pulse is set to be 1.75 volts to eliminate noise or spike, and to take the oscillation at its beginning. As soon as the amplitude of the pulse is identified (crossed threshold), the microcontroller will ignore the signal for $450 \mathrm{~ms}$ to prevent any false identification due to the presence of premature pulse "overshoot" due to oscillation. Hence, this algorithm can only detect pulse rate which is less than 133 beats per minute $(60 / 0.45=133)$. The time delay is chosen to be $450 \mathrm{~ms}$ as shown in Figure 5 from [10]. This is equal to half the average period of a heart pulse for a normal healthy person which is typically 0.9 seconds. The program then takes the average of five time intervals so that the heart rate will be as accurate as possible.

\section{Diastolic Pressure Measurement Method}

After the heart- rate is determined, the program enters this mode where it is still sampling the signal at every 40 millisecond.

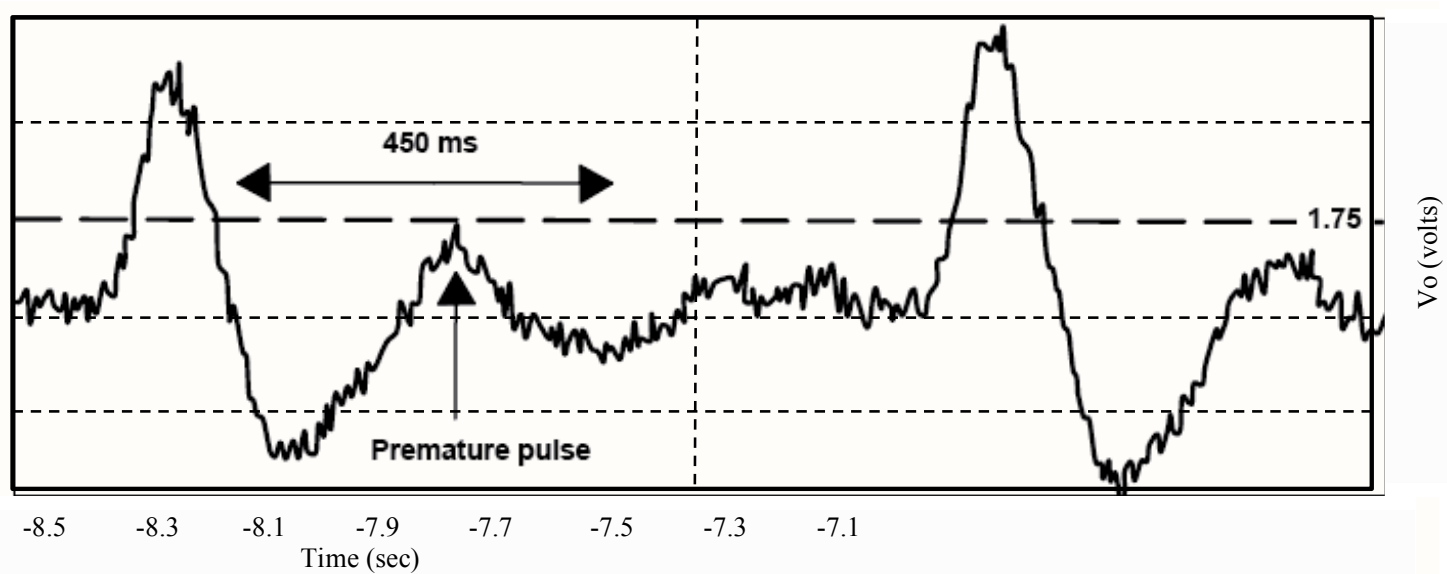

Figure 5. Zoom- in view of a pulse. 
We then define the threshold for the diastolic pressure. While the cuff is deflating, at some point before the pressure reaches diastolic pressure, the amplitude of the oscillation will decrease. To determine the diastolic pressure, we record the DC value (from AN1) at the point when the amplitude of the oscillation decreases to below the threshold voltage. This is done by looking at the time interval of 1.2 seconds. If the AC waveform does not go above the threshold in 1.2 seconds, it means the amplitude of the oscillation is actually below the threshold. The DC value recorded then will be the value that represents the diastolic pressure.

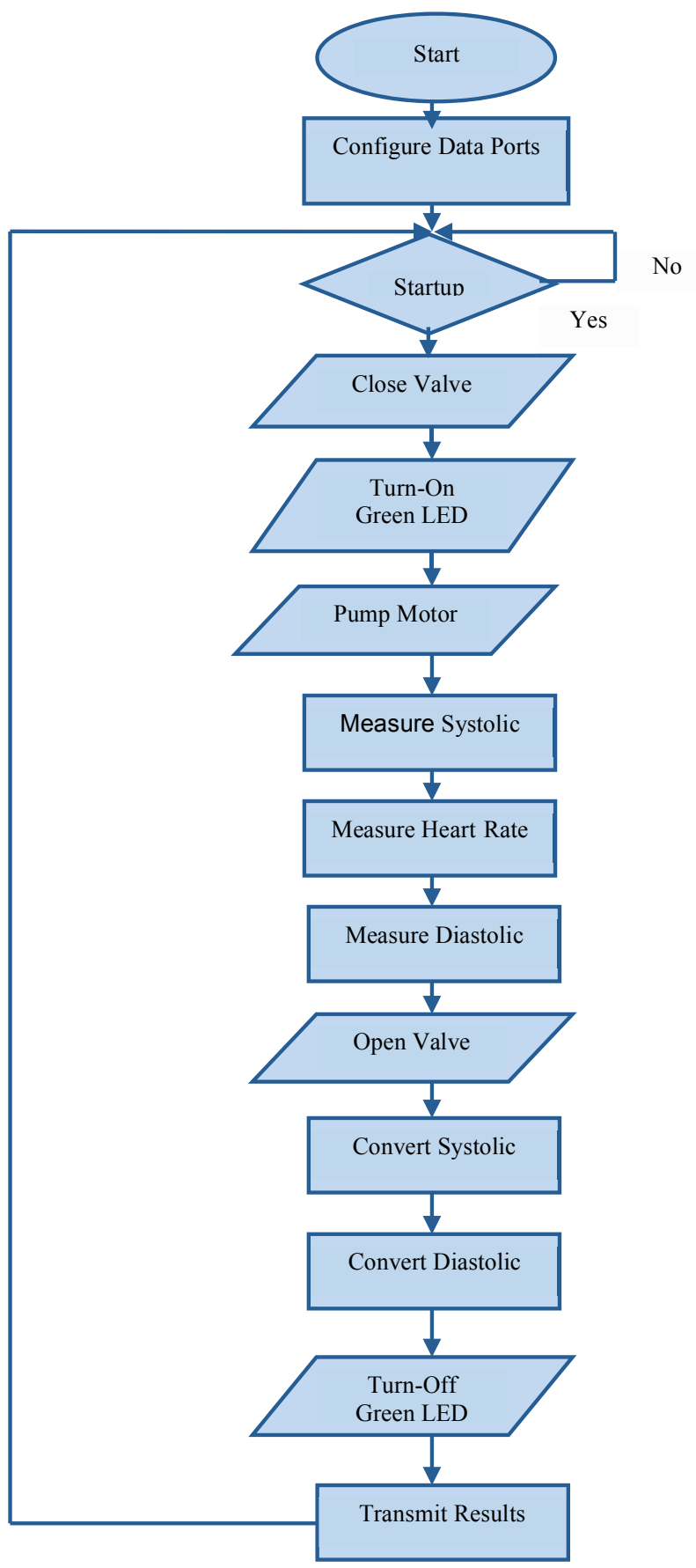

Figure 6. The microcontroller program flowchart.
After this method finishes, the microcontroller is programmed to send the measurements on its UART (universal asynchronous receiver transmitter) to the Bluetooth module. Figure 6 depicts a flow chart of the microcontroller program.

\section{The Mobile AppliCATION}

\section{A. Application Software Development}

Our main objective here was to develop a mobile application that is capable of communicating with our blood pressure monitoring device remotely over Bluetooth technology. We target to achieve two main ideas in our application: the first was to build a mobile application that has the ability to remotely start the blood pressure measurement operation on the device. While the second was to be able to received data from the blood pressure monitor device, process it and display it in a convenient manner on the screen.

To accomplish both tasks, we decided to build a Windows Mobile application that can be run on any Microsoft Windows CE device [8]. The basic requirement for our application is simply a mobile device or pocket PC that runs Windows Mobile 6 or later, as its operating system. Our development tool was Microsoft Visual Studio 2005 with service pack-1. In order, to develop windows mobile application in Microsoft Visual Studio the Windows Mobile 6 SDK (software development kit) had to be installed also. The earlier tool allows you to develop Windows Mobile applications in any .Net programming language. The basic idea behind .Net programming is the .Net framework which includes plenty of predefined libraries and functions that facilitate the development of a well-managed and more sophisticated programs and applications. In building our application, we also used the .Net compact framework version 3.5 in addition to the Windows Embedded Source Tools for Bluetooth Technology, from MSDN shared resources to control the Bluetooth radio in the mobile.

In developing our mobile application, we decided to adopt $\mathrm{CH}$ as our programming language.

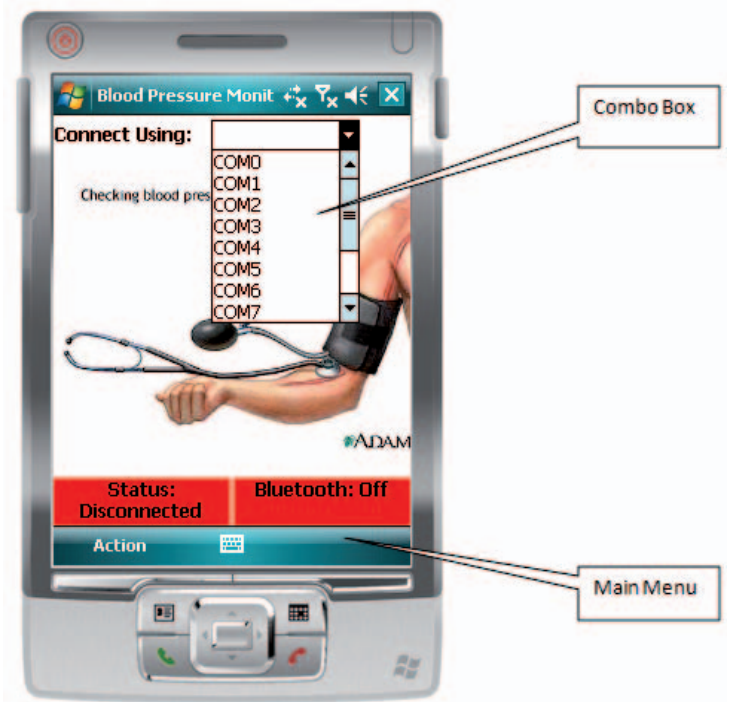

Figure 7. Selecting COM port in mobile application 
It is worth mentioning that our software application can still run on earlier versions of Windows CE such as Windows Mobile 5.0 under one condition which is installing the required service pack and .Net compact framework version 3.5 or later. In our mobile application, we chose communication using Bluetooth via the serial port profile, and there were various reasons behind our choice. To start with, the microcontroller in our device communicates with the Bluetooth module serially using UART serial communication port. This implies that our Bluetooth module supports the serial profile. In addition, Windows Mobile has a nice feature which is COM Port emulation; this enables virtual $\mathrm{COM}$ ports to be created over radio frequency channels. In other words, once Bluetooth is pair to another Bluetooth device, you can map it to a virtual COM port and communicate with it using the serial port profile.

\section{B. Application Software Operation}

.When the application software is first run, the mobile device will not be connected to the BP monitoring device, thus the connection status will be set to "disconnect". Also, it will check the status of the Bluetooth radio to see whether it is turned-on or turned-off and display the correct status. The user has to select a proper COM port to establish the connection as shown in Fig. 7. The program will then check the status of the Bluetooth radio. If the Bluetooth radio is turned-off, it will be turned-on automatically in order to establish the connection. Moreover, there will be a separate thread from the main program thread that will be running in the background, which will continuously monitor and check for changes in the status of the Bluetooth radio and connection status. This thread will be able to detect changes made by the program or by the user external to the application software, from the mobile device operating system. If any change has been detected, this thread will invoke another function on the main program thread to make the corresponding updates on the user interface elements. For instance, if Bluetooth was turned-on from the mobile itself, the Bluetooth status will change from $O F F$ to $O N$, and the color of this label will change from red to green, and a notification message will appear on the screen.

Now once the program is running and a proper connection has been established, the user will be capable of measuring the BP and heart-rate using the BP monitor device. This would be accomplished by selecting the "Measure Blood Pressure" button under Action menu. A progress bar will be shown on the screen indicating operation progress as shown in Fig. 8. The user will not be able to use any function on the main application unless the measurement process has ended. This is a form that shows that the BP measuring process is in progress. It will show up once the measurement process is started. It contains a picture box that contains an image of a dummy ECG signal, and the picture box is animated to move from left to right, from one edge of the form to the other.

It will automatically disappear once the measurement process has ended and data is received from the $\mathrm{BP}$ device by the mobile. A measurement panel is used to display the results (readings) received from the $\mathrm{BP}$ monitor device. This panel will show up in the application immediately displaying the results once data

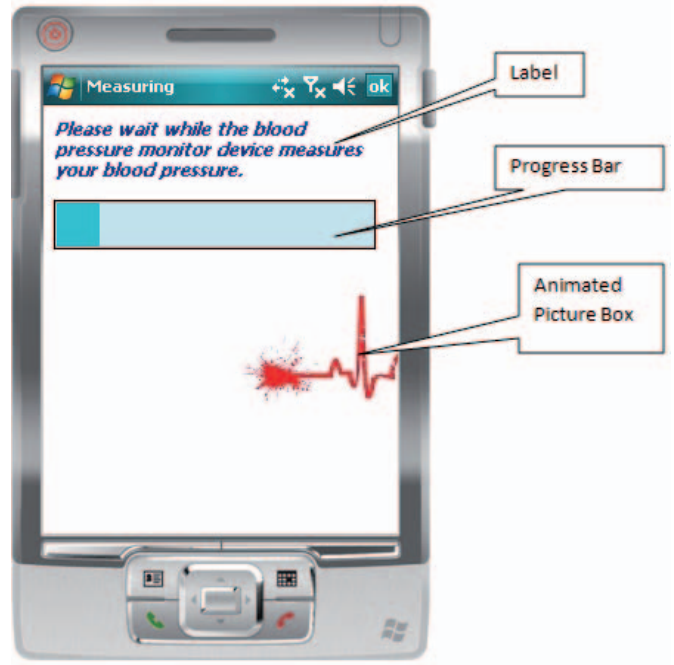

Figure 8. Measuring form

is received as shown in Fig. 9. The panel consists of several items as follows: a) the SBP and DBP readings in $\mathrm{mmHg}$. b) the heart-rate reading in pulses/min. c) picture box that shows a dummy heart image. d) a close-form button that will allow the user to close the panel and return back to main form.

\section{Analysis of MEASUREMENTS AND RESUlts}

In order to verify the reliability and accuracy of the readings obtained using our BP monitoring system, we compared them to a set of readings obtained by a commercially available device that has already been calibrated and tested for reliability. We chose the Visomat handy BP monitor found in the market as our reference. We compared results obtained by our device to results using the reference, based on the assumption that the reference readings are nominal and error free. We fixed all variables in our testing environment so that

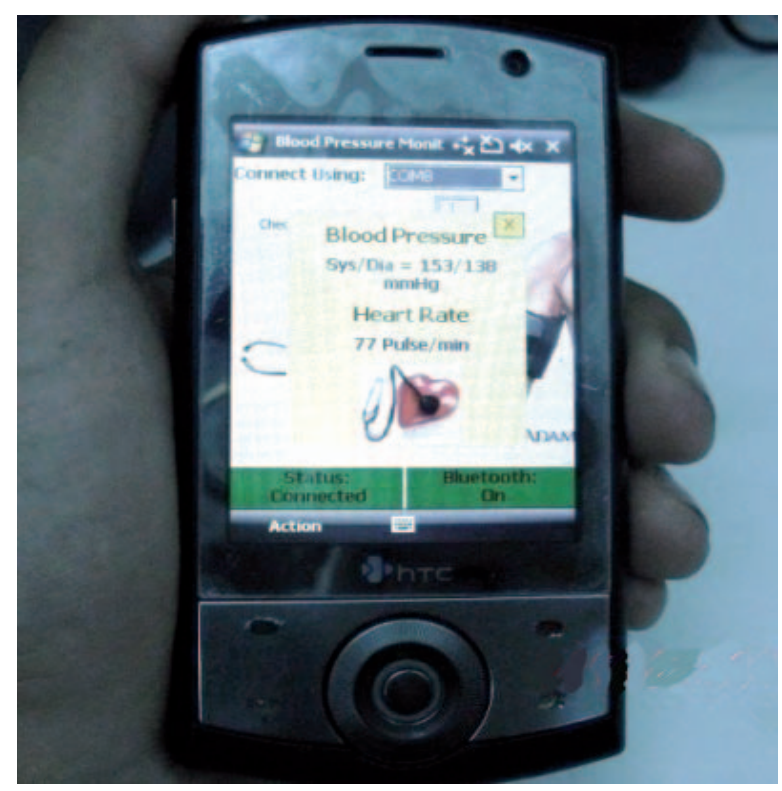

Figure 9. Mobile phone displaying BP and HR measurements. 
the only changing variable is the measuring device.

Our statistical analysis was done by taking 50 readings of BP and HR for a human subject using our device and the reference device. Ten of the measurement values taken are listed below in Table I. representing a sample of the corresponding measurements of DBP, SBP, and HR.

TABLE I.

MEAN VALUES AND ERRORS

\begin{tabular}{|c|c|c|c|c|c|c|}
\hline \multirow[b]{2}{*}{$\begin{array}{c}\text { Read. } \\
\text { No. }\end{array}$} & \multicolumn{3}{|c|}{ Our System } & \multicolumn{3}{|c|}{ Reference } \\
\hline & $\begin{array}{c}\text { SBP } \\
\mathrm{mmHg}\end{array}$ & $\begin{array}{c}\text { DBP } \\
\text { mmHg }\end{array}$ & $\begin{array}{c}\text { HR } \\
\text { Beat/ } \\
\text { min }\end{array}$ & $\begin{array}{c}\text { SBP } \\
\text { mmHg }\end{array}$ & $\begin{array}{c}\text { DBP } \\
\mathrm{mmHg}\end{array}$ & $\begin{array}{c}\text { HR } \\
\text { Beat/ } \\
\text { min }\end{array}$ \\
\hline 1 & 152 & 75 & 96 & 141 & 84 & 93 \\
\hline 2 & 150 & 82 & 99 & 141 & 84 & 93 \\
\hline 3 & 140 & 78 & 94 & 147 & 78 & 94 \\
\hline 4 & 157 & 74 & 101 & 118 & 67 & 103 \\
\hline 5 & 148 & 68 & 93 & 144 & 69 & 89 \\
\hline 6 & 150 & 68 & 95 & 147 & 52 & 93 \\
\hline 7 & 143 & 73 & 95 & 137 & 88 & 98 \\
\hline 8 & 145 & 68 & 92 & 147 & 82 & 94 \\
\hline 9 & 140 & 69 & 93 & 148 & 85 & 95 \\
\hline 10 & 136 & 70 & 96 & 147 & 64 & 87 \\
\hline Mean & 146.1 & 72.5 & 95.4 & 141.7 & 75.3 & 93.9 \\
\hline $\begin{array}{l}\text { Abs. } \\
\text { Error }\end{array}$ & 4.4 & 2.8 & 1.5 & & & \\
\hline $\begin{array}{c}\text { Error } \\
(\%) \\
\end{array}$ & 3.01 & 3.86 & 1.6 & & & \\
\hline
\end{tabular}

We then conducted statistical analysis of the deviations between the two (measurement error) to compare the performance of our BP telemedicine system to the reference, and also to classify the statistical distribution of the measurement-error. This will allow us to establish an expected tolerance and to structure a form of a confidence interval for the set of obtained measurements. We show in Fig. 10 the three histograms we have generated that represent the distributions of the errors (deviations of our device from the reference) of DBP, SBP, and HR measurements. We can fairly assume that the errors follow Normal (Gaussian) distribution functions with statistical means of zero for each. This has the practical inference that our over-all performance (repeated measurements) is very reliable because the average of the error in measurements is zero.

To further analyze and characterize the reliability of our BP smart-phone-based system, we listed the calculated mean value from the tabulated sample of measurements of Table I. for each type of reading obtained by the reference device and ours. Table I. illustrates the results of analysis, which proves the effectiveness of our versatile BP measurement system. Finally, we show in Fig. 11 a 3-D plot of the confidence interval for any triple-valued measurement of DBP-SBPHR obtained using our system. It is apparent from the plot that the HR measurement has the smallest deviation (best confidence interval) because most of the error is within the vicinity of the zero-plane (light blue color), whereas the DBP and SBP average of confidence interval is mostly contained within the \pm 10 planes.
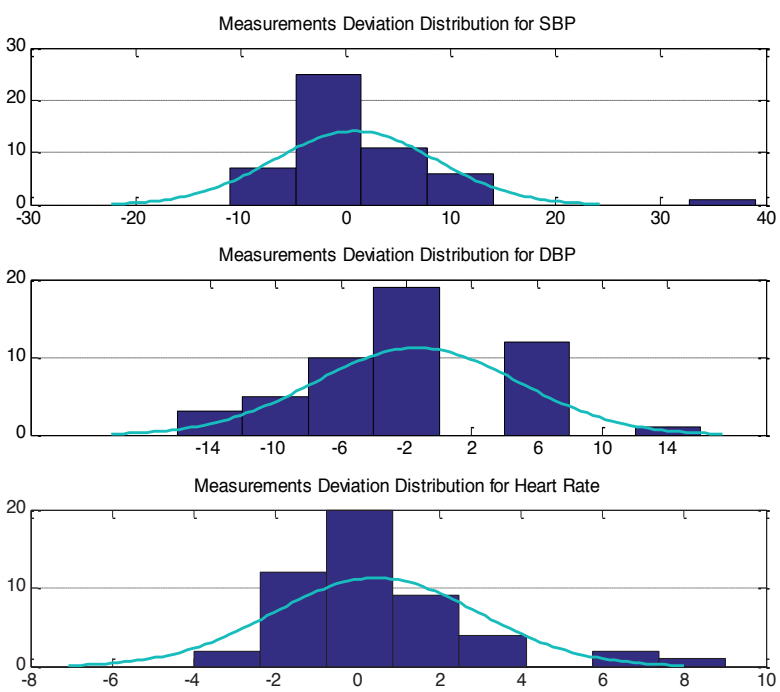

Figure 10. Histograms of measurements error

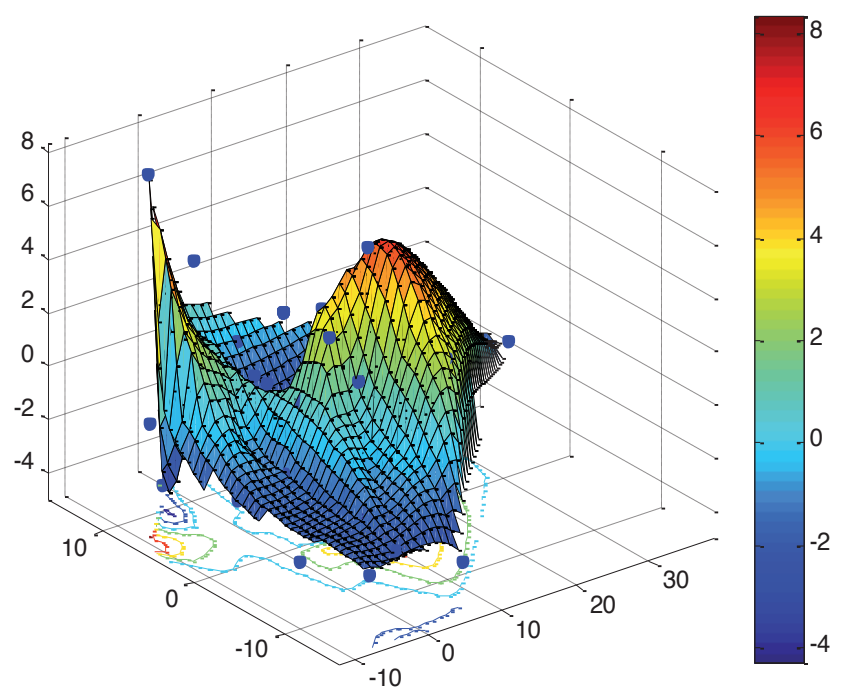

Figure 11. A 3-D (surface) plot of confidence interval

\section{CONCLUSION}

In this paper, we have presented a detailed structure and theory of a reliable low-cost telemedicine wireless monitoring system that can measure blood pressure (BP) and heart-rate (HR). We have relied on commercially available components and a Windows Mobile platform smart-phone to implement our system. The main distinguishing feature of our system design is the utilization of a smart-phone to control the hardware subsystem of our device and to display and store measurement results that can be accumulated locally or stored on a remote server in a database. The use of the smart-phone built-in components has reduced overall hardware cost. We also developed a custom-made mobile application software with a practical interface which 
emphasized user operation simplicity. In addition, this system can be viewed as a proof-of-concept to demonstrate its applicability in telemedicine, and to help in combating the most common chronic medical problem of chronic high BP. That is because this universal smartphone-based monitoring system can be deployed in many versatile settings including hospitals, nursing-homes, health-centers, and homes for its ease of use and convenience. Moreover, the reliability and accuracy of our system was investigated and demonstrated through detailed statistical analysis of a sample of collected measurements, which has produced confidence intervals and reference performance metrics.

\section{REFERENCES}

[1] P. Puska, S. Mendis, D. Porter, "Chronic Diseas - Key Risk factors," World Health Organization (WHO), 2003

[2] P. Kanavos, Jan Östergren, High Blood Pressure and Health Policy: where we are and where we need to go next, Report, 2007.

[3] S. Ahmad, S. Chen, K. Soueidan, I. Batkin, M. Bolic, H. Dajani, V. Z. Groza, "Electrocardiogram-assisted blood pressure estimation," in IEEE Trans. Biomed. Eng., vol. 59, no. 3, pp. 608618, Mar. 2012. http://dx.doi.org/10.1109/TBME.2011.2180019

[4] L. A. Geddes, Handbook of blood pressure measurements. Totowa, NJ: Humana Press Inc., 1991. http://dx.doi.org/10.1007/ 978-1-4684-7170-0

[5] Y. Choi, J. Krause, H. Seo, K. Kaptain, K. Chung, Telemedicine in the USA: Standardization through information management and technical application, IEEE Communications. Magazine, vol. 44(4), 2006, 41-48. http://dx.doi.org/10.1109/MCOM. 2006.1632648

[6] A. Tahat, "Mobile messaging services-based personal electrocardiogram monitoring system," International Journal of Telemedicine and Applications, vol. 2009, pp. 1-7, June 2009. http://dx.doi.org/10.1155/2009/859232

[7] International Telecommunications Union (ITU), The World ICT Statistics 2013, http://www.itu.int//TU-D/ict/statistics/ , April 2013.

[8] A. Tahat, A. Sacca, Y. Kheetan, "Design of an integrated mobile system to measure blood-pressure," IEEE 18th Symposium on Communications and Vehicular Technology in the Benelux (SCVT), November 2011, Belgium, pp. 1-6 .

[9] Freescale Semiconductor, Integrated Silicon pressure sensor (MPX5050) datasheet, Chandler, Arizona, USA, Mach 2010.

[10] Freescale Semiconductor, Digital Blood Pressure Meter (AN1571) application note, Chandler, Arizona, USA, May 2005.
[11] D. Kammer, G McNutt, B. Senese, Bluetooth Application Developer's Guide. Rockland, MA, Syngress Publishing, 2002.

[12] R. Bouhenguel, I. Mahgoub, M. Ilyas, "Bluetooth Security in Wearable Computing Applications," in Proc. International Symposium on High Capacity Optical Networks and Enabling Technologies, Nov. 2008, pp. 182-186 http://dx.doi.org/10.1109/ HONET.2008.4810232

\section{AUTHORS}

Dr. Ashraf Tahat received the B.S., M.S., and Ph.D. degrees in Electrical Engineering from the Illinois Institute of Technology (IIT), Chicago, Illinois, USA, in 1992, 1993, and 2002, respectively. He is an Associate Professor with the Department of Communications Engineering at PSUT in Amman, Jordan where he was the Department Chairman. $\mathrm{He}$ is currently a Visiting Professor in the Department of Electrical and Computer Engineering at McGill University in Montreal, Quebec, Canada, working on digital signal processing for modern communications systems such as LTE-A. In 2002, he worked at the ECE Department, IIT, as a Post-Doctoral Fellow. From 1998 to 2000, he was with 3Com Corporation working on voice-band PC modems. In 1997, he joined Lucent Technologies (AT\&T), Inc. (currently Alcatel-Lucent) as a consultant Research Engineer to assist in the development of ADSL modems. Dr. Tahat is a senior member of IEEE. (e-mail: aat@ieee.org).

Yazeed Kheetan is a radio frequency (RF) engineer with Ericsson-Jordan conducting drive-test measurements for site verification purposes. He received the BS degree in communications engineering from Princess Sumaya University for technology (PSUT) in Amman, Jordan in 2011. He also worked at Orange-Jordan as an engineer with the mobile access network department.

Anwar Sacca received the BS degree in computer engineering from Princess Sumaya University for technology (PSUT) in Amman, Jordan in 2011. He is currently working at Elite Technologies JLT company in Dubai, UAE as a branch manager.

This article is an extended and modified version of a paper presented at the IEEE 18th Symposium on Communications and Vehicular Technology in the Benelux (SCVT), held in November 2011, in Ghent, Belgium. Submitted 22 April 2013. Published as re-submitted by the authors 15 September 2013. 